\title{
Arginine68 is an essential residue for the C-terminal cleavage of human Atg8 family proteins
}

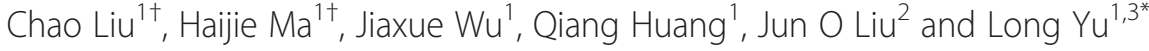

\begin{abstract}
Background: Autophagy is a conserved cellular process that degrades and recycles cytoplasmic components via a lysosomal pathway. The phosphatidylethanolamine (PE)-conjugation of the Atg8 protein plays an important role in the yeast autophagy process. In humans, six Atg8 homologs, including MAP1LC3A, MAP1LC3B, MAP1LC3C (refer to LC3A, LC3B, and LC3C hereafter), GABARAP, GABARAPL1, and GABARAPL2 have been reported. All of them can be conjugated to PE through a ubiquitin-like conjugation system, and be located to autophagosomes.

Results: In this study, we found 3 new alternative splicing isoforms in LC3B, GABARAP, and GABARAPL1, (designated as LC3B-a, GABARAP-a and GABARAPL1-a, respectively). None of them can go through the PEconjugation process and be located to autophagosomes. Interestingly, compared with LC3B, LC3B-a has a single amino acid (Arg68) deletion due to the NAGNAG alternative splicing in intron 3. Through structural simulations, we found that the C-terminal tail of LC3B-a is less mobile than that of LC3B, thus affecting its C-terminal cleavage by human ATG4 family proteins. Furthermore, we found that Arg68 is an essential residue facilitating the interaction between human Atg8 family proteins and ATG4B by forming a salt bridge with Asp171 of ATG4B. Depletion of this salt bridge reduces autophagosomes formation and autophagic flux under both normal and nutrition starvation conditions.
\end{abstract}

Conclusions: These results suggest Arg68 is an essential residue for the C-terminal cleavage of Atg8 family proteins during the autophagy process.

Keywords: Atg8, MAP1LC3B, Autophagy, Alternative splicing

\section{Background}

Macroautophagy (hereafter referred to as autophagy) is an evolutionarily conserved cellular process which degrades and recycles cytoplasmic components through the de novo synthesized membrane system and lysosome $[1,2]$. The activity of autophagy is important for cellular homeostasis, cell survival, development, and aging [3]. Deregulated autophagy results in many physiological defects, including liver injury, muscular disorder, neurodegeneration, pathogen infections and cancer [4-9].

In response to stress conditions, such as starvation, autophagy can be up-regulated. Cytoplasmic components are

\footnotetext{
* Correspondence: longyu@fudan.edu.cn

${ }^{\dagger}$ Equal contributors

'State Key Laboratory of Genetic Engineering; Institute of Genetics; School of Life Sciences, Fudan University, Shanghai 200433, China

${ }^{3}$ Institute of Biomedical Sciences, Fudan University, Shanghai 200433, China

Full list of author information is available at the end of the article
}

sequestered into an expanding phagophore, which finally encloses to form double membrane vesicles, named autophagosomes. Autophagosomes are transported and fused with lysosomes, then the inner membrane and cargo can be digested by multiple enzymes existing in lysosomes. The resulting small molecules are finally transported out of the lysosomes for recycling [1].

In the past 16 years, molecular genetic studies in yeast have identified at least 32 autophagy-related genes (ATG) functioning in different steps of autophagy $[10,11]$. Among them, the ubiquitin-like protein Atg8 is the only protein that could bind to all autophagic membrane structures, thus it is commonly used as a marker to trace autophagosomes [12]. Before binding with autophagic membrane structures, newly synthesized Atg8 protein (pro-Atg8) is processed by a cysteine protease, Atg4. Thus the C-terminus is cleaved and the conserved glycine residue is exposed, forming Atg8-I molecules [13]. Then Atg8-I protein is covalently conjugated with 
PE through a ubiquitin-like conjugation system, including E1-like enzyme Atg7 [14,15], E2-like enzyme Atg3 [16], and E3-like enzyme Atg12-Atg5-Atg16 complex [17]. The PE-modified Atg8 (Atg8-II protein) is relocated to the autophagic membrane structures and mediates the membrane tethering, expanding, and cargo recognition [18-21].

In humans, Atg8 homologs comprise of six members: LC3A, LC3B, LC3C [22-25], GABARAP [24,26], GABARAPL1/ATG8L [27] and GABARAPL2/GATE-16 $[24,28]$. The conserved post-translational modification process on these Atg8 family proteins is essential for their biological function during the autophagy process [23,24,29-31]. To fully explore the family members of human Atg8 homologs, we analysed their alternative splicing patterns and found new isoforms for LC3B, GABARAP, and GABARAPL1, designated as LC3B-a, GABARAP-a and GABARAPL1-a respectively. Interestingly, comparing to LC3B, LC3B-a lacks only one amino acid (Arg68) due to the NAGNAG alternative splicing [32], and has severely impaired C-terminal cleavage efficiency by ATG4B, one of the major members of the human ATG4 family proteins [29]. Furthermore, we found that Arg68 was also an essential residue for the interaction between Atg8 homologs and Atg4B in human. Mutation of Arg68 in human Atg8 family proteins would severely decrease their C-terminal cleavage efficiency, thus affecting their autophagic localization and autophagic flux. Taken together, we report the identification and characterization of an essential residue for the autophagic activity of human Atg8 family proteins, which provides potential targets to regulate the autophagy activity in human diseases.

\section{Results}

Identification of new isoforms in human Atg8 family proteins

Alternative mRNA splicing generates a diverse range of mature RNAs, and potentially expands the cellular protein repertoire [33]. Thus, one gene can be translated to multiple proteins with distinct biological functions. To fully explore the members in human Atg8 family proteins, we searched their alternative splicing patterns from the Alternative Splicing and Transcript Diversity (ASTD) database which has been integrated in Ensembl (http://www.ensembl.org) [34]. As shown in Figure 1A, we found new mRNA transcripts in $L C 3 B, G A B A R A P$, and GABARAPL1. We named their alternative transcripts as $L C 3 B-a$, GABARAP- $a$, and GABARAPL1-a, respectively [GenBank: JN663879, JN663880, and JN663881]. Specifically, the isoform of GABARAP-a or GABARAPL1-a adopt different 3' ends, resulting the missing of the conserved glycine necessary for ATG4 cleavage and down-stream processing [35]. LC3B has two tandem 3' splice acceptor sites (TAGAAG) in intron 3 (Figure 1A). Using the distal splice site (AAG) would remove AAG as part of the exon 4, thus resulting in the loss of Arg68 after translation.

To verify the existence of these transcripts, we cloned these transcripts from a HeLa cDNA library. The sequencing result for each transcript is shown in Additional file 1: Figure S1. We also amplified each transcript by allele-specific PCR from multiple human tissue cDNA libraries. Primer specificity for LC3B and LC3B-a were tested by using $\mathrm{LC} 3 \mathrm{~B}$ and $\mathrm{LC} 3 \mathrm{~B}-\mathrm{a}$ plasmids as templates. As shown in Additional file 1: Figure S2, the primers we designed can distinguish both transcripts specifically. As shown in Figure 1B, all three newly identified transcripts exist in human tissues. While $L C 3 B$, $L C 3 B-a$, and GABARAP express ubiquitously in human tissues, GABARAP- $a$ and GABARAPL1- $a$ have tissue specificities. Furthermore, the expressed sequence tags (EST) supporting the existence of each transcript were shown in Additional file 1: Table S1.

\section{Three new isoforms could neither undergo post- translational modification nor be localized to autophagosomes}

The removal of AAG as part of exon 4 results in the loss of Arg68 in the amino acid sequence of LC3B-a (Additional file 1: Figure S3). GABARAP-a and GABARAPL1-a lack the highly conserved $\beta 4$ strand and Gly120 (Additional file 1: Figure S3), an indispensable amino acid for their C-terminal cleavage by Atg4 [35]. Thus, these two isoforms may not be processed by human Atg4 family proteins. To explore the post-translational modification patterns of these new isoforms, we first fused these proteins with myc-tag at the $\mathrm{N}$ termini and his-tag at the $\mathrm{C}$-termini. Bands detected by anti-myc antibody show all forms of molecules after modification, including the pro form (non-cleaved form), the -I form (cleaved form) and the -II form (cleaved and PE conjugated form). Bands detected by anti-his antibody show only the pro form (non-cleaved form). As shown in Figure 2A, the C-terminal cleavages of LC3B-a, GABARAP-a, and GABARAPL1-a are all severely impaired. Large amounts of pro-form accumulate in the cell lysates.

Next, we fused GFP protein to the N-terminal of these proteins and examined their intracellular localization in HeLa cells under starvation conditions. As shown in Figure $2 \mathrm{~B}$, except for the three newly found members, all these proteins form puncta in the cytoplasm and colocalize to autophagosomal marker p62 [36]. In contrast, none of the new isoforms could be localized to autophagosomes but only ubiquitously disperses in the cytosol, even under starvation conditions.

These results show that the newly found isoforms could hardly be cleaved by human Atg4 family proteins, 


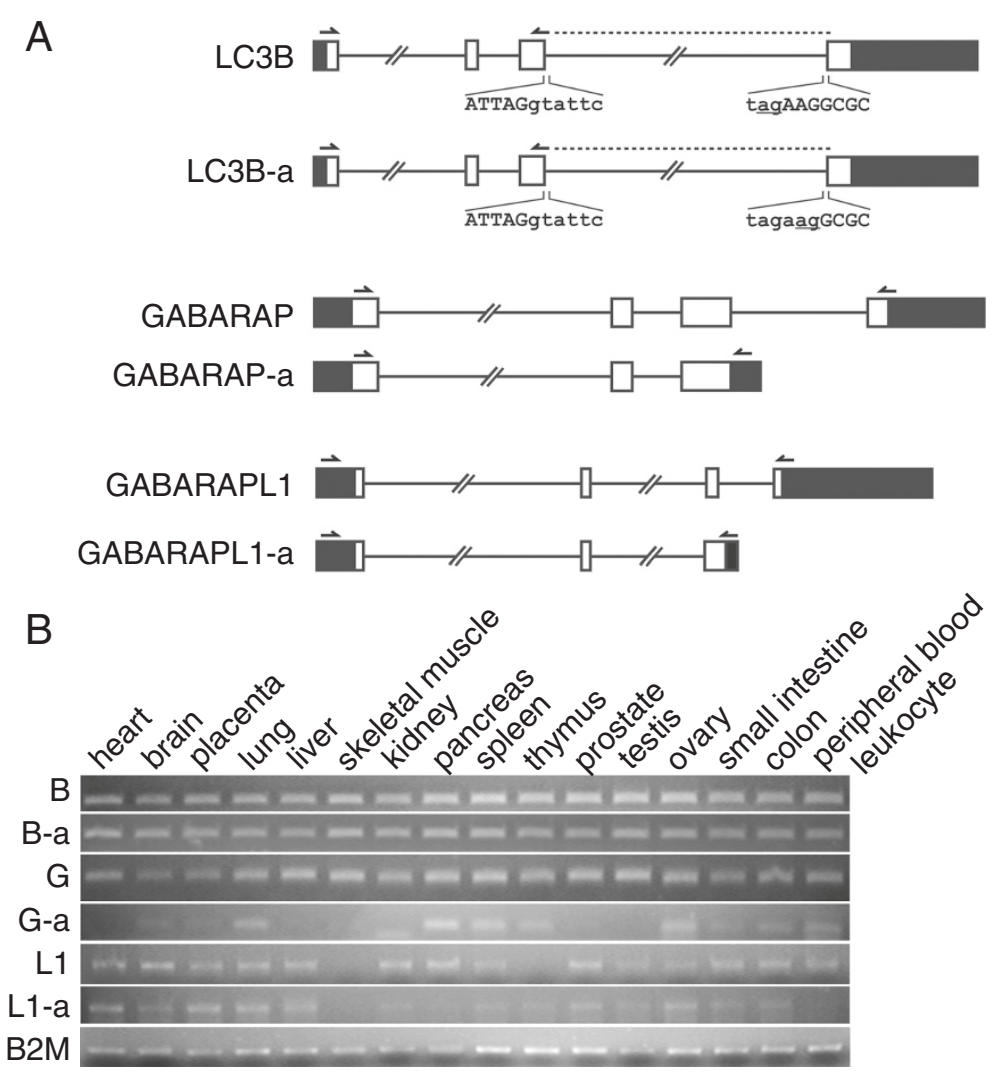

Figure 1 Alternative splicing patterns and expressions of human Atg8 homologs. A. Schematic diagram of the alternative splicing patterns of $\angle C 3 B, G A B A R A P$ and GABARAPL1 mRNA. White and black boxes represent the protein-coding or non-coding region, respectively. Straight lines represent introns. Nucleotide sequences at the intron 3 splicing sites are shown. Splicing acceptors are underlined. Arrows show the positions of the corresponding allele specific primers. B. Expression of each transcript in multiple human tissues. Semi-quantitative PCR for each transcript was performed on human multiple-tissue CDNA panels. The expression of housekeeping gene B2M was analysed as loading control. B, LC3B; B-a, LC3B-a; G, GABARAP; G-a, GABARAP-a; L1, GABARAPL1; L1-a, GABARAPL1-a.

thus affecting their following modification process and autophagosomal localization.

\section{Conformational changes resulting from the loss of Arg68 inhibit the cleavage of LC3B-a by ATG4}

The C-termini of GABARAP-a and GABARAPL1-a could not be cleaved by human Atg4 family proteins maybe due to the lack of the conserved Gly120, which is indispensable for C-terminal cleavage [35]. Interestingly, the only difference between the two isoforms of LC3B is the presence (in LC3B) or absence (in LC3B-a) of Arg68, suggesting that Arg68 may play an important role in the C-terminal cleavage of LC3B. To investigate the reason why the cleavage efficiency of LC3B-a from human ATG4 family proteins is severely affected, we first compared the interaction between ATG4B and these two isoforms. As shown in Additional file 1: Figure S4, both isoforms could be pulled down by ATG4B, suggesting that the interactions between ATG4B and these two isoforms are intact, and the low cleavage efficiency maybe due to other reasons.
Then, we focused on the structure of human Atg8 family proteins. The structure of Atg8 [37], rat LC3 [38], GABARAP [39,40], GABARAPL2 [41] have been reported. They all contain 2 helices at the $\mathrm{N}$-terminus and a conserved ubiquitin fold at the C-terminus. Arg68 is a conserved amino acid and located on the $\alpha 3$ helix of the ubiquitin fold (Additional file 1: Figure S3 and Figure 3B).As the $\alpha 3$ helix is the core of the ubiquitin fold, the deletion of Arg68 may affect the ubiquitin fold of LC3B-a. Taking advantage of the known crystal structures of three LC3B homologs: rat LC3 [38], GABARAP [40], and GABARAPL2 [41], we performed molecular modeling to assess the possible structural difference between LC3B and LC3B-a. The C-terminal fragments, including the conserved Gly120 in all three structures are disordered, and atomic coordinates of certain C-terminal residues are not available. Given the significant sequence conservation between LC3B and rat LC3, it was expected that the C-terminal tail of LC3B is also disordered in solution. We performed nanosecond molecular dynamics (MD) simulations to determine the motion 


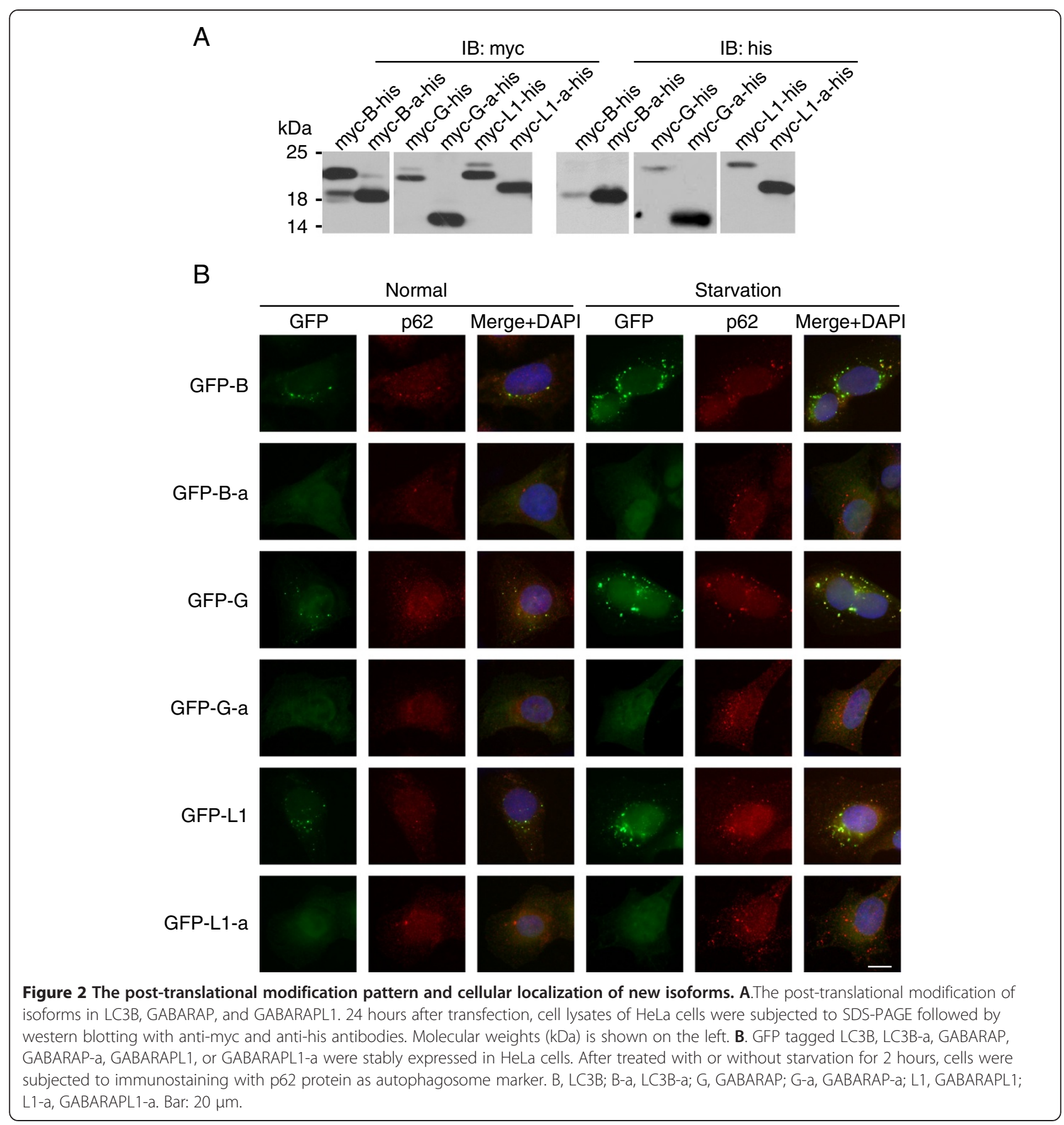

states of the C-terminal segments of LC3B and LC3B-a in solution. We constructed the initial structures of both isoforms with the homology modeling method using the crystal structures of LC3B homologs as templates (see Methods). Two protein-water systems for MD simulations of LC3B and LC3B-a were then built (Figure 3A). Next, 13-ns MD simulations for each system were carried out. Since the protocols for the simulations of the two isoforms are the same, the resultant models of the two proteins can be compared to each other.
The modeling of LC3B showed that its C-terminal segment is quite flexible and disordered in solution, in agreement with those structures reported in the LC3B homologs $[38,41]$ In the simulations, the C-terminal segment of about ten amino acids (Asn116 to Val125) were always projected into the aqueous phase and did not possess any stable conformation. In contrast, the main body of the protein is relatively compacted and conformationally rigid (Figure 3B). The simulation of LC3B-a reveals that its $\mathrm{C}$-terminal conformation is far 


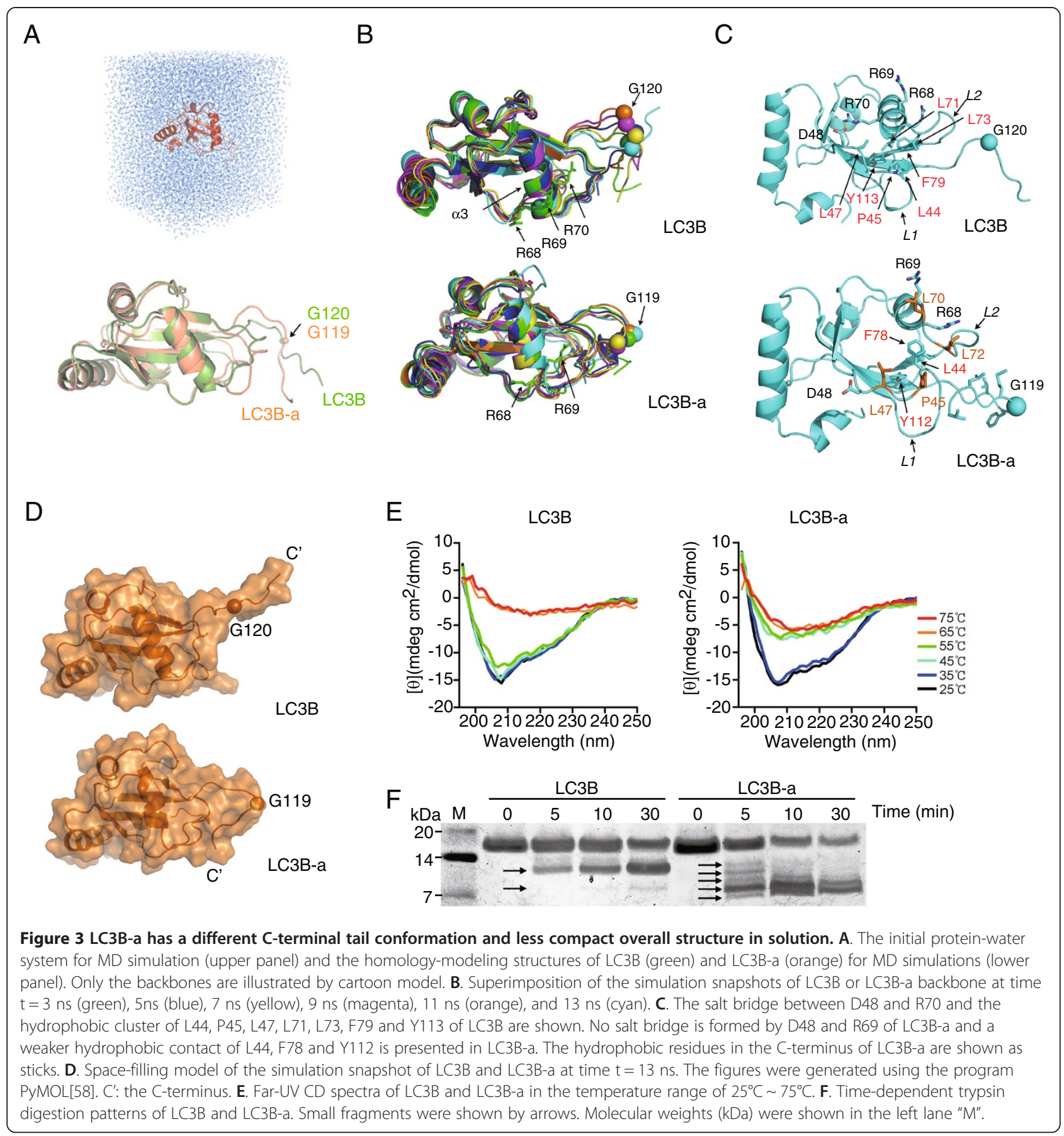

less mobile. It is bound to the main body of the protein in a helical form, as illustrated by simulation snapshots of the backbone conformation (Figure 3B).

The absence of Arg68 disrupts a salt bridge between the side chains of Arg70 and Asp48 which is also an important internal interaction in LC3 [41]. Also, the absence of Arg68 leads to a reduction of one rotation in the $\alpha 3$ helix, which is important in stabilizing the interaction between loops L1 (a.a. 38-50) and L2 (a.a. 71-79) (Figure $3 \mathrm{C}$ ) and maintaining the ubiquitin fold structure.
The absence of one rotation in the $\alpha 3$ helix takes the two loops apart, disrupting the hydrophobic cluster formed by Leu44, Pro45, Leu47, Leu71, Leu73, Phe79, and Tyr113 in LC3B, leaving a weaker interaction among only three residues, Leu44, Phe78 and Tyr112 in LC3B-a (Figure 3C). Together, these changes significantly weakens the dynamic interaction between loops L1 and L2, allowing the exposed hydrophobic groove between them to accommodate the hydrophobic side chain of Thr117, Phe118, Met120, Leu122 and Val124 in the C- 
terminus of LC3B-a. Thus, the C-terminal tail of LC3B-a may attach to the main protein body, shielding the hydrophobic chain from solvent and preventing the cleavage by the scissile peptide bond of human ATG4 family proteins, as shown by the space-filling models of the proteins (Figure 3D).

The MD simulation revealed that the intramolecular interaction in LC3B-a is weaker than that of LCB. To confirm this, we analysed their far-UV circular dichroism spectra under different temperatures. As shown in Figure 3E, the spectra of these two isoforms were similar at room temperature, indicating that the overall structure of LC3B-a did not change significantly from that of LC3B. However, as the temperature increased, LC3B-a lost its characteristic minimum ellipticity of the $\alpha$ helix at $208 \mathrm{~nm}$ at $45^{\circ} \mathrm{C}$. In contrast, this signal of LC3B did not change until the temperature reached $65^{\circ} \mathrm{C}$. These results suggest that $\mathrm{LC} 3 \mathrm{~B}-\mathrm{a}$ is thermally less stable than LC3B.

In addition, we also used trypsin digestion of LC3B and LC3B-a to assess their conformational flexibility. As shown in Figure 3F, LC3B was cleaved into one main short fragment within the first 5 minutes and the second short fragment appeared after 30 minutes of trypsin digestion. In contrast, LC3B-a was quickly cleaved to at least five small fragments in the first 5 minutes. Thus, LC3B's main body had a more compact and stable structure in solution than LC3B-a, despite its more flexible $\mathrm{C}$-terminus. These results suggest that the intramolecular interactions of LC3B-a is weaker than that of LC3B and the C-terminus of LC3B-a may be accommodated into the exposed hydrophobic groove, which inhibits the cleavage by ATG4 and inhibits the whole posttranslational modification process.

\section{Arg68 is essential for the interaction between human Atg8 family proteins and ATG4B}

Three continuously conserved arginines (Arg68, Arg69, and Arg70) are located together in the $\alpha 3$ helix of LC3B. Thus, to determine whether these arginine residues are essential for their autophagic function, we mutated each of them to alanine and analysed their post-translational modification patterns by western blotting. To our surprise, as shown in Figure 4A, the R68A mutation had significantly accumulated pro-form of LC3B, while the modification patterns of R69A and R70A mutants were almost the same as the wild type protein (Figure 4A), suggesting that Arg68, not Arg69 or Arg70, is essential for the C-terminal cleavage of LC3B. This effect of Arg68 in LC3B is also conserved in other homologs of human Atg8 proteins. As shown in Figure 4B, mutation of the corresponding Arg68 of LC3B (Arg68 of LC3A, Arg74 of LC3C, Arg65 of GABARAP and GABARAPL1) to alanine also results in the accumulation of the pro- form of each protein, suggesting that the biological function of Arg68 is conserved among human Atg8 homologs.

The three continuously conserved arginines (Arg68, Arg69, and Arg70) are located together in the $\alpha 3$ helix of LC3B, which may form a local positively charged area and serve as a potential protein-protein interaction sites. As mentioned above, only Arg68 can affect the cleavage of human Atg8 family proteins. Thus, we wondered whether Arg68 was an essential residue for the interaction with human ATG4 family proteins. Since the crystal structure of the complex between rat LC3 and human ATG4B has been solved [42], we took advantage of these structures to examine the amino acids likely to be involved in the protein-protein interactions. As shown in Additional file 1: Figure S5, the side chain of Arg68 points to the interface of these two proteins, forming a salt bridge with Asp171 of ATG4B. We then compared the binding affinity of LC3B and LC3B-R68A with ATG4B in the presence of $1 \mathrm{mM}$ PMSF, which could inhibit the cysteine protease activity of ATG4B. As shown in Figure $4 \mathrm{C}$ and $4 \mathrm{D}$, the interaction between these two proteins was decreased when mutating Arg68 of LC3B or Asp171 of ATG4B, indicating that the salt bridge between Arg68 and Asp171 is important for the LC3B-ATG4B interaction. We also performed a time-dependent in vitro cleavage assay using purified GST-ATG4B, GST-ATG4B-D171A, LC3B, and LC3BR68A proteins. As shown in Figure 4E, wild type ATG4B and LC3B could finish their cleavage within 10 minutes, leaving only the up-shifted -I form protein. However, when the salt bridge was destroyed, there were still large amounts of pro-LC3B left in the reaction mixture after 20 minutes. Thus, the mutation of Arg68 or Asp171 of ATG4B impaired the cleavage efficiency significantly.

\section{Arg68 is essential for the autophagosome localization and autophagic flux.}

Arg68 mutation did not abolish the PE-conjugation (Figure 4B, long exposure), indicating that R68A mutant proteins can go through the post-translational modification. However, the functional importance of Arg68 in mammalian cells was still unknown. Thus, we stably expressed GFP-LC3B or GFP-LC3B-R68A in HeLa cells, and counted their autophagosomal localization under normal culture condition or starvation condition. As shown in Figure 5A, GFP-LC3B-R68A showed a significant decrease of autophagosome puncta number in both conditions. Furthermore, after the starvation stimulation, the increase of GFP-LC3B-R68A puncta was much lower than that of GFP-LC3B, indicating that the LC3B-R68A mutant protein has lower autophagic activity in mammalian cells. 


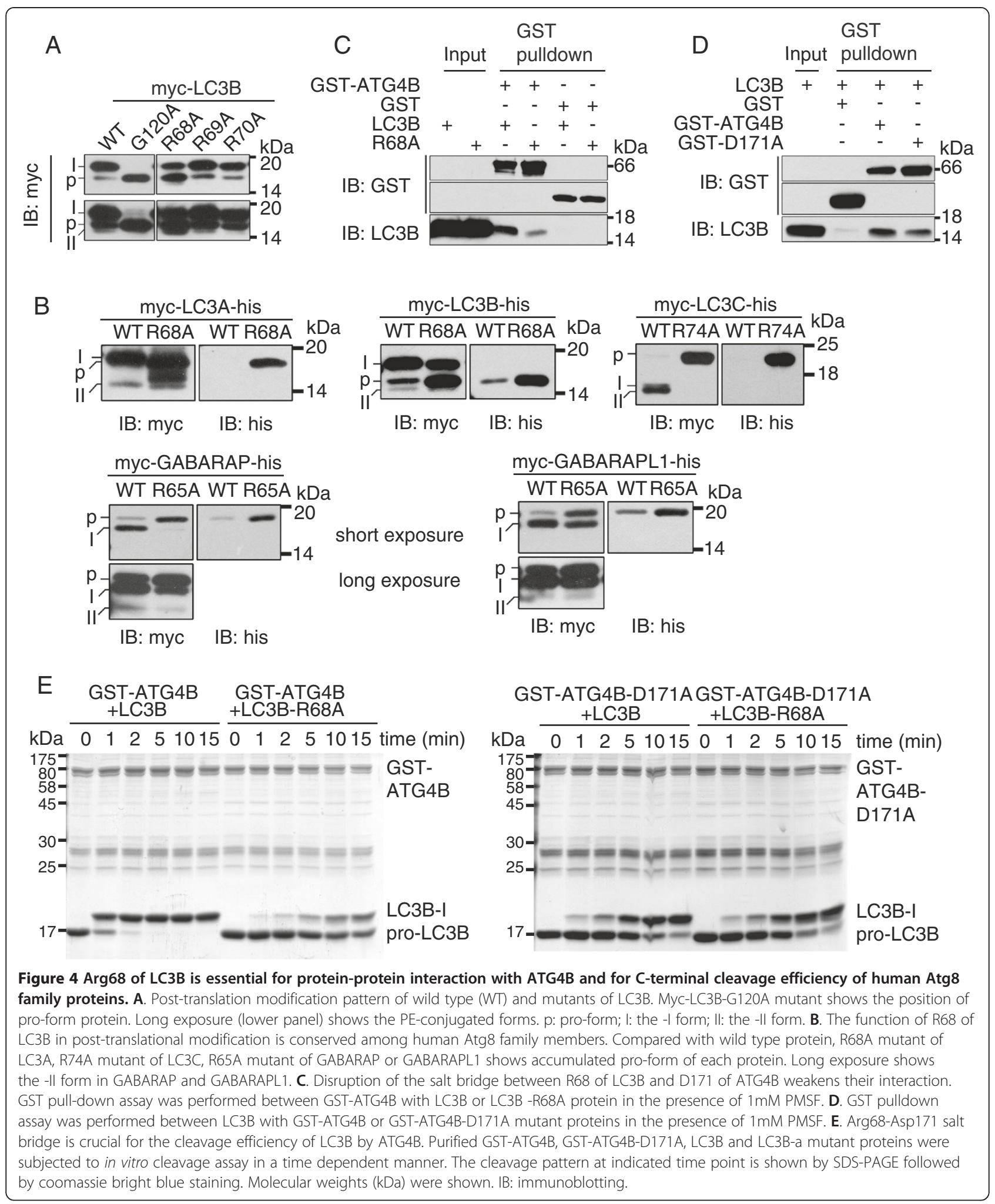

We also measured the autophagic flux by monitoring the LC3B turnover. HeLa cells expressing either mycLC3B or myc-LC3B-R68A were supplied ammonium chloride $\left(\mathrm{NH}_{4} \mathrm{Cl}\right)$, a lysosomotropic reagent which attenuates the acidification of the lysosome and inhibits the degradation of LC3B-II, resulting in the accumulation of LC3B-II. Thus, the difference of LC3B-II ratio (LC3B-II/ total LC3B) between the samples in the 


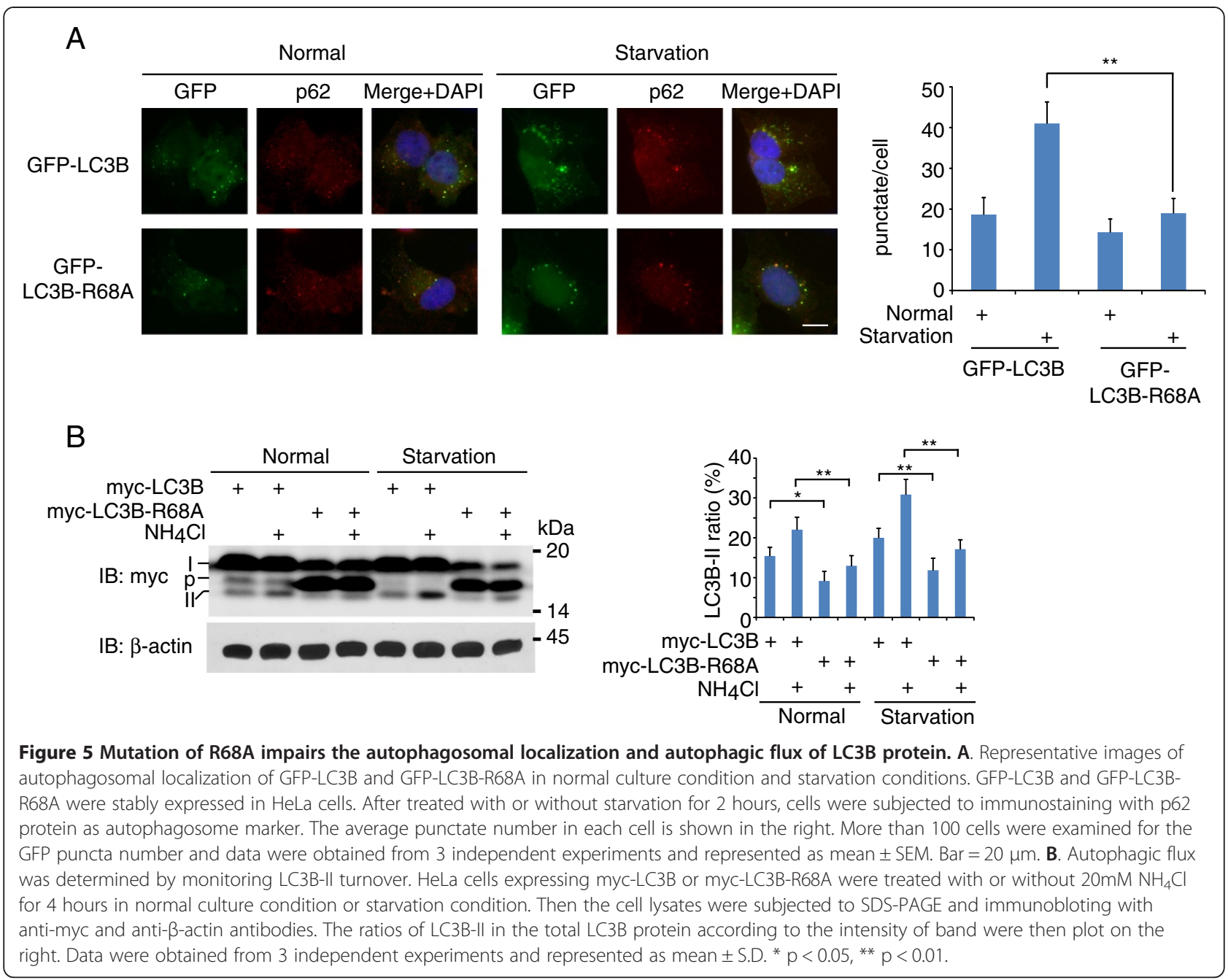

presence or absence of $\mathrm{NH}_{4} \mathrm{Cl}$ represents the amount of LC3B delivered and degraded in lysosomes. As shown in Figure $5 \mathrm{~B}$, the LC3B-II ratio increase of wild type LC3B is significantly higher than that of LC3B-R68A mutant protein in both normal and starvation conditions, indicating the autophagic flux of LC3B-R68A is lower than that of wild type LC3B.

Both the results of autophagosomal localization and autophagic flux suggest that the LC3B-R68A mutant has lower autophagic activity in mammalian cells. As mentioned above, Arg68 of LC3B or the equivalent arginines in other homologs are key amino acids for protein-protein interaction between human Atg8 homologs and ATG4B. The mutation of Arg68 may decrease the cleavage efficiency by ATG4B, thus affecting its function in the autophagy process.

\section{Discussion}

The post-translational processing of LC3B play essential roles in autophagy, especially in the formation of autophagosomes. In our previous work, we identified three members of the human LC3 family, LC3A, LC3B, and LC3C [22]. In that paper, we showed that LC3B could not undergo post-translational modification as LC3A and LC3C. In contrast, Tanida et al. reported that the C-terminus of LC3B could be cleaved to expose the conserved Gly120 for further modification [35]. To verify our observations, we sequenced the plasmids used in our previous work and found that some of our $L C 3 B$ plasmids used in the previous paper were $L C 3 B-a$, the new alternative splicing isoform of $L C 3 B$ reported in this paper. This explained our different result with that reported by Tanida et al. about the post-translational modification of LC3B.

Alternative splicing is an important mechanism for controlling gene expression and creating the great proteomic complexity from a limited number of genes [43]. The NAGNAG alternative splicing which happens in the human $L C 3 B$ gene is actually widespread in the human genome, occurring in $30 \%$ of human genes [32]. The NAGNAG motif is also conserved in $L C 3 B$ and $L C 3 C$ genes in different species, including Homo sapiens, Pan troglodytes, Canis 
lupus familiaris, Bos Taurus, as well as the $L C 3 B$ gene of Rattus norvegicus and $L C 3 C$ gene of Gallus gallus. Protein isoforms resulting from the NAGNAG motifs have also been shown to display functional diversity, such as signaling activity [44], cellular localization [45], DNA binding activity [46], protein-protein interaction affinity [47]. Here, we report that LC3B-a has distinct post-translational modification patterns with that of LC3B. Since LC3B-a can interact with ATGB but cannot be cleaved by ATG4B, LC3B-a may have inhibitory effect of human Atg8 family proteins cleavage by ATG4B; this needs further investigation. Atg8 and its homologs were found to be the only protein associating with all autophagic related structures [12,23]. Its functions were largely unknown for a long time until recently Nakatogawa et al. reported that Atg8 is responsible for the autophagic membrane tethering and hemifusion [20] and cargo receptor recognition [48]. The structures of Atg8 homologs are important in understanding their functional mechanisms. Amar et al. have identified two sites which are required for autophagy by mutational analyses $[49,50]$. Phe77 and Phe79 of Atg8, equivalents of Phe80 and Leu82 in LC3B are part of the Atg4 recognition site, and Tyr49 and Leu50 are important for the downstream lipidation steps. Through alternative splicing analysis, we have found Arg68 as a new protein interaction site with ATG4B, which is important for the initial post-translational modification process of human Atg8 family proteins.

\section{Conclusions}

The alternative splicing pattern analysis of human Atg8 family proteins revealed that the Arg68 of LC3B and the corresponding arginine in other human Atg8 family members is an essential residue for the post-translational modification of these ubiquitin-like molecules. In addition to its role in stabilizing the ubiquitin-core structure, Arg68 also plays an important role in the interaction between Atg8 family proteins and ATG4B proteins.

\section{Methods}

\section{Allele-specific semi-quantification PCR amplification and} sequencing

Allele-specific PCR primers were designed according to the method of Simsek et al. [51] and listed in Additional file 1: Table S2. Human MTC Panel I (Cat. No. 636742) and Human MTC Panel II (Cat. No. 636743) (Clontech) were used as PCR templates and the semi-quantification PCR was performed according to the manufacturer's instructions. PCR products were then resolved on $2 \%$ agarose-EB gels.

For DNA sequencing, we used ABI PRISM Big Dye Terminator Cycle Sequencing V2.0 Ready Reaction Kit and the ABI PRISM 3730 DNA analyzer (Applied Biosystems, Foster City, Calif., USA) according to the manufacturer's instructions.

\section{Plasmids and antibodies}

Genes were cloned to pCMV-myc, pGEX-4T-1, and pEGFP-c1 vectors according to the standard protocol. The mutants of each gene were generated by using the QuikChange site-directed mutagenesis kit (Stratagene). Primers for cloning and mutation are listed in Additional file 1: Table S3. Anti-myc, anti-his and anti-GST monoclonal antibodies were purchased from Upstate. Anti-p62 antibody was purchased from abcam (ab56416). Rabbit anti-LC3B antibody was raised against human LC3B full length protein.

\section{Cell culture, transfection and Western blot}

HeLa cell was cultured in Dulbecco's Modified Eagle Medium with $10 \%$ fetal bovine serum. For starvation induction, cells were washed three times with PBS and incubated with Earle's balanced salt solution (ESBB; Invitrogen) at $37^{\circ} \mathrm{C}$ for indicated time. For $\mathrm{NH}_{4} \mathrm{Cl}$ inhibition, $20 \mathrm{mM} \mathrm{NH}_{4} \mathrm{Cl}$ was added to medium for 4 hours.

Cells were transfected with lipofectamine (Invitrogen) according to the manufacturer's instructions. 24 hours after transfection, cells were subjected to further analysis. Stable cell line preparation was performed following standard protocols.

\section{Protein expression, purification and pulldown assay}

The GST fusion proteins were expressed in BL21(DE3) E. coli. cells and purified using a glutathione-Sepharose $4 \mathrm{~B}$ column (Novagen) followed by cleavage of thrombin (Novagen) if needed. All purified proteins were dialysis against PBS prior to use or storage.

$2 \mu \mathrm{g}$ GST or GST-ATG4B protein were incubated with $5 \mu \mathrm{g}$ LC3B or LC3B mutant proteins with Glutathione Sepharose $4 \mathrm{~B}$ beads (GE Healthcare) at $4^{\circ} \mathrm{C}$ for 2 hours in the presence of $1 \mathrm{mM}$ PMSF with rotation. Then the beads were thoroughly washed in ice-cold PBS for 5 times and then boiled in the SDS sample buffer for further analysis.

\section{Immunofluorescence confocal microscopy and quantification of the GFP-LC3 puncta}

Cells stably expressing indicated GFP fusion proteins were treated with normal or starvation conditions. Then they were washed with ice-cold PBS 3 times and fixed in $4 \%$ paraformaldehyde at room temperature for 5 minutes. Then the cells were treated with $0.5 \%$ Triton X-100 in PBS at room tem-perature for 5 minutes. Cell samples were then blocked with $8 \%$ goat serum in PBS for 30 minutes and incubated with the primary antibody at room temperature for one hour. After three washing with PBS, samples were then incubated with the second antibody at room temperature for 30 minutes and then co-stained with 
DAPI for one minute. After 3 times of final washing by PBS, cells were then mounted and observed by using a $63 \mathrm{x}$ Oil immersion objective using a ZEISS LSM 710 Laser Scanning Confocal Microscope. The quantification of GFP puncta was performed by Image J version 1.38 (National Institutes of Health). More than 100 cells were quantified in each sample.

\section{Constructions of initial structures of LC3B and LC3B-a for molecular simulations}

The crystal structure of rat LC3 (Protein Data Bank code: 1UGM) [38] was employed as the template for homology modeling to construct the initial structure of LC3B, as the amino acid sequence of rat LC3B is almost identical to that of LC3B. The initial structure of LC3B was built by directly submitting the sequence and structural template to the Swiss-MODEL server (http:// swissmodel.expasy.org) [52]. This homology modeling based on 1UGM gave only the initial atomic coordinates of amino acids 5-117, because 1UGM lacks the coordinates of other amino acids. Since this study was intended to investigate the entire $\mathrm{C}$-terminal region of the protein, we constructed the initial coordinates for amino acids 3-4 and 118-120 according to the crystal structure of another homologous protein GATE-16 by directly employing the backbone $(\phi, \phi)$ angles of the corresponding amino acids (Protein Data Bank code: 1EQ6) [41]. Finally, the initial coordinates of remaining amino acids were determined by randomly setting their backbone rotational angles $(\phi, \psi)$ without any atomic overlap. With the initial structure, we performed MD simulations for LC3B as described below. Then, we used the simulation snapshot structure of LC3B at 1 ns to construct the initial structure of LC3B-a via the Swiss-MODEL server.

\section{MD simulations with explicit solvent representation}

MD simulations were performed with the all-hydrogen force fields OPLS-AA $[53,54]$ using the program suite GROMACS 3.1.4. [55,56]. The TIP4P water model48 was employed. To construct a simulation system, initial all-hydrogen structure of LC3B (or LC3B-a) was merged into a rectangular box of water, in which the water molecules were placed randomly without atomic overlap. The thickness of the water layer between the protein and the closest box-boundary is $\sim 1.4 \mathrm{~nm}$. As such, the simulation system for an isoform contains $\sim 9,700$ water molecules for the simulated water density of $\sim 1 \mathrm{~g} / \mathrm{cm}^{3}$. Finally, counterpart ions were placed into the box to make the system neutral. For all the simulations, we used the ensemble of constant number of molecules, pressure, and temperature (N-P-T ensemble), with the temperature held at $300 \mathrm{~K}$ and the pressure at $1 \mathrm{~atm}$. In the simulations, the Berendsen temperature coupling method [57] was used with a coupling constant of
0.1 ps. Cutoff distance for van der Waals force was set to $1.0 \mathrm{~nm}$. Electrostatic forces were calculated by the particle mesh Ewald (PME) method50 with a cutoff of $1.0 \mathrm{~nm}$ for real-space interactions, and the reciprocalspace interactions were computed on a $0.12 \mathrm{~nm}$ grid with fourth-order spline interpolation. The simulations were run under periodical boundary conditions using a time step of 2 fs. Two parallel simulations were performed for LC3B- $\alpha$ and LC3B- $\beta$. The period for each simulation run is $13 \mathrm{~ns}$. Such a simulation time-scale is usually needed for the C-terminal segment which initial coordinates of 5 amino acids were randomly assigned to adjust to its conformations to the equilibrium states.

\section{Circular dichroism spectroscopy}

Circular dichroism (CD) spectra were recorded at $\mathrm{pH}$ 7.0 in a Jasco J-810 spectropolarimeter equipped with a Peltier temperature control unit. The concentrations of proteins were $40 \mu \mathrm{M}$ for the far-UV measurements (185-260 nm, light path $1 \mathrm{~mm})$. The spectra obtained were the average of 4 scans. The absorbance from the buffer control was subtracted from the protein spectra. Thermal transition curves $\left(25^{\circ} \mathrm{C}\right.$ to $\left.75^{\circ} \mathrm{C}\right)$ were determined by monitoring the decrease in ellipticity at 222 $\mathrm{nm}$ at a scanning rate of $40^{\circ} \mathrm{C} / \mathrm{h}$ using a $1 \mathrm{~mm}$ pathlength cell.

\section{Trypsin digestion}

An aliquot of $50 \mathrm{ng}$ trypsin was added to $20 \mu \mathrm{L}$ PBS $(\mathrm{pH}=7.4)$ containing $1 \mu \mathrm{g}$ LC3B or LC3B-a, followed by incubation in $37^{\circ} \mathrm{C}$ water bath. Aliquots of the reaction mixture were taken out at the indicated time interval, quenched with $5 \mu \mathrm{L} 5 \mathrm{X}$ gel loading buffer and heated at the $98^{\circ} \mathrm{C}$ water bath for $8 \mathrm{~min}$. The samples were resolved on $4 \% \sim 12 \%$ NuPAGE $^{\circ}$ Novex Bis-Tris gels that were stained with silver.

\section{In vitro ATG4B cleavage assay}

$6 \mu \mathrm{g}$ of GST-ATG4B or GST-ATG4B-D171A proteins were mixed with $12 \mu \mathrm{g}$ LC3B or LC3B-R68A proteins in $120 \mu \mathrm{l}$ reaction volume of PBS, followed by incubation in $30^{\circ} \mathrm{C}$ water bath. At the time of $0,1,2,5,10$ minutes, $20 \mu \mathrm{l}$ reaction mixtures were subjected to sampling preparation. All the samples are subjected to SDS-PAGE and Commassie Blue Staining.

\section{Autophagic flux determination}

$1 \mu \mathrm{g}$ construct of myc-LC3B or myc-LC3B-R68A was transfected into 6-well plate of HeLa cells. 24 hours after transfection, cells were treated with normal or starvation conditions in the presence or absence of $20 \mathrm{mM} \mathrm{NH} 4 \mathrm{Cl}$ for 4 hours. Cell lysates were then subjected to SDSPAGE and immunobloting with anti-myc antibody. Fluorescence signals were obtained by Bio-Rad 
ChemiDoc ${ }^{\mathrm{Th}} \mathrm{XRS}+$ System and bands signals were quantified by Image Lab ${ }^{\text {Tw }}$ Software version 2.0 .

\section{Additional file}

Additional file 1: Table S1. Gene Bank accession numbers of ESTs. Table S2. Allele specific primers for each transcript. Table S3. Primers used in this paper for cloning and mutagenesis. Figure S1. The sequencing results of $\angle C 3 B-a$, GABARAP- $a$ and GABARAPL1- $a$. Figure S2. Primer specificity test for LC3B and LC3B-a transcripts. Figure S3. Alignment of human Atg8 family proteins. Figure S4. The interaction between $L C 3 B-a$ and ATG4B is intact as that of LC3B. Figure S5. Cartoon representation of human ATG4B-rat LC3B complex.

\section{Abbreviations}

Phosphatidylethanolamine: PE; Expressed sequence tags: EST;

Molecular dynamics: MD

\section{Competing interests}

The authors declare that they have no competing interests.

\section{Authors' contributions}

$\mathrm{CL}, \mathrm{HM}$, and JW performed most of the experiments. QH performed the MD simulation. JOL, $\mathrm{LY}$ designed the experiments and analysed data. $\mathrm{CL}$ wrote the paper. All authors read and approved the final manuscript.

\section{Acknowledgements}

We thank Dr. Jianping Ding (Institute of Biochemistry and Cell Biology, Chinese Academy of Sciences, Shanghai, China) and Dr. Yanhui Xu (Institute of Biomedical Sciences, Fudan University, Shanghai, China) for faithful advices of LC3B structure analysis, Dr. Xianmei Yang for initial paper draft preparation, Yefang Gong and Yunmin Xu for technical assistance. The authors are grateful to Henry Kuang and Zhonghao Wang for proof reading. This work was supported by the National 973 program of China (2004CB518605), the National 863 project of China (2006AA020501), the National Key Sci-Tech Special Project of China (2008ZX10002-020), the Project of the Shanghai Municipal Science and Technology Commission (03dz14086) and the National Natural Science foundation of China (30024001, 30771188).

\section{Author details}

${ }^{1}$ State Key Laboratory of Genetic Engineering; Institute of Genetics; School of Life Sciences, Fudan University, Shanghai 200433, China. ${ }^{2}$ Departments of Pharmacology and Molecular Sciences and Oncology, Johns Hopkins University School of Medicine, Baltimore, MD 21205, USA. ${ }^{3}$ Institute of Biomedical Sciences, Fudan University, Shanghai 200433, China.

Received: 6 November 2012 Accepted: 15 May 2013

Published: 30 May 2013

\section{References}

1. Klionsky DJ, Emr SD: Autophagy as a regulated pathway of cellular degradation. Science 2000, 290(5497):1717-1721.

2. Yang Z, Klionsky DJ: Eaten alive: a history of macroautophagy. Nat Cell Biol 2010, 12(9):814-822.

3. Shintani T, Klionsky DJ: Autophagy in health and disease: A double-edged sword. Science 2004, 306(5698):990-995.

4. Donohue TM Jr: Autophagy and ethanol-induced liver injury. World J Gastroenterol 2009, 15(10):1178-1185.

5. Nishino I: Autophagic vacuolar myopathies. Curr Neurol Neurosci Rep 2003, 3(1):64-69.

6. Cherra SJ 3rd, Dagda RK, Chu CT: Review: autophagy and neurodegeneration: survival at a cost? Neuropathol Appl Neurobiol 2010, 36(2):125-132.

7. Huang J, Brumell JH: Autophagy in immunity against intracellular bacteria. Curr Top Microbiol Immunol 2009, 335:189-215.

8. Li Y, Zhang J, Chen X, Liu T, He W, Chen Y, Zeng X: Molecular Machinery of Autophagy and Its Implication in Cancer. Am J Med Sci 2012, 343:155-161.
9. Huang J, Klionsky DJ: Autophagy and human disease. Cell Cycle 2007, 6(15):1837-1849.

10. Klionsky DJ, Cregg JM, Dunn WA Jr, Emr SD, Sakai Y, Sandoval IV, Sibirny A, Subramani S, Thumm M, Veenhuis M: A unified nomenclature for yeast autophagy-related genes. Dev Cell 2003, 5:539-545.

11. Yang Z, Klionsky DJ: Mammalian autophagy: core molecular machinery and signaling regulation. Curr Opin Cell Biol 2010, 22(2):124-131.

12. Yorimitsu T, Klionsky DJ: Autophagy: molecular machinery for self-eating Cell Death Differ 2005, 12(Suppl 2):1542-1552.

13. Kirisako T, Ichimura Y, Okada H, Kabeya Y, Mizushima N, Yoshimori T, Ohsumi M, Takao T, Noda T, Ohsumi Y: The reversible modification regulates the membrane-binding state of Apg8/Aut7 essential for autophagy and the cytoplasm to vacuole targeting pathway. I Cell Biol 2000, 151(2):263-276.

14. Kim J, Dalton VM, Eggerton KP, Scott SV, Klionsky DJ: Apg7p/Cvt2p is required for the cytoplasm-to-vacuole targeting, macroautophagy, and peroxisome degradation pathways. Mol Biol Cell 1999, 10(5):1337-1351.

15. Tanida I, Mizushima N, Kiyooka M, Ohsumi M, Ueno T, Ohsumi Y, Kominami E: Apg7p/Cvt2p: a novel protein-activating enzyme essential for autophagy. Mol Biol Cell 1999, 10(5):1367-1379.

16. Schlumpberger $M$, Schaeffeler $E$, Straub $M$, Bredschneider $M$, Wolf DH Thumm M: AUT1, a gene essential for autophagocytosis in the yeast Saccharomyces cerevisiae. J Bacteriol 1997, 179(4):1068-1076.

17. Mizushima N, Sugita H, Yoshimori T, Ohsumi Y: A new protein conjugation system in human. J Biol Chem 1998, 273(51):33889-33892.

18. Ichimura $Y$, Imamura $Y$, Emoto $K$, Umeda M, Noda T, Ohsumi Y: In vivo and in vitro reconstitution of Atg8 conjugation essential for autophagy. J Biol Chem 2004, 279(39):40584-40592.

19. Sou YS, Tanida I, Komatsu M, Ueno T, Kominami E: Phosphatidylserine in addition to phosphatidylethanolamine is an in vitro target of the mammalian Atg8 modifiers, LC3, GABARAP, and GATE-16. J Biol Chem 2006, 281(6):3017-3024.

20. Nakatogawa $H$, Ichimura $Y$, Ohsumi $Y$ : Atg8, a ubiquitin-like protein required for autophagosome formation, mediates membrane tethering and hemifusion. Cell 2007, 130(1):165-178.

21. Xie Z, Nair U, Klionsky DJ: Atg8 controls phagophore expansion during autophagosome formation. Mol Biol Cell 2008, 19(8):3290-3298.

22. He H, Dang Y, Dai F, Guo Z, Wu J, She X, Pei Y, Chen Y, Ling W, Wu C, et al: Post-translational modifications of three members of the human MAP1LC3 family and detection of a novel type of modification for MAP1LC3B. J Biol Chem 2003, 278(31):29278-29287.

23. Kabeya Y, Mizushima N, Ueno T, Yamamoto A, Kirisako T, Noda T, Kominami E, Ohsumi Y, Yoshimori T: LC3, a mammalian homologue of yeast Apg8p, is localized in autophagosome membranes after processing. EMBO J 2000, 19:5720-5728.

24. Kabeya Y, Mizushima N, Yamamoto A, Oshitani-Okamoto S, Ohsumi Y, Yoshimori T: LC3, GABARAP and GATE16 localize to autophagosomal membrane depending on form-II formation. J Cell Sci 2004, 117(13):2805-2812.

25. Mann SS, Hammarback JA: Molecular characterization of light chain. Part 3. J Biol Chem 1994, 269(15):11492-11497.

26. Wang $H$, Bedford FK, Brandon NJ, Moss SJ, Olsen RW: GABAA-receptorassociated protein links GABAA receptors and the cytoskeleton. Nature 1999, 397:69-72.

27. Tanida I, Sou YS, Minematsu-lkeguchi N, Ueno T, Kominami E: Atg8L/Apg8L is the fourth mammalian modifier of mammalian Atg8 conjugation mediated by human Atg4B, Atg7 and Atg3. FEBS J 2006, 273(11):2553-2562.

28. Sagiv $Y$, Legesse-Miller A, Porat A, Elazar Z: GATE-16, a membrane transport modulator, interacts with NSF and the Golgi v-SNARE GOS-28. EMBO J 2000, 19:1494-1504.

29. Hemelaar J, Lelyveld VS, Kessler BM, Ploegh HL: A single protease, Apg4B, is specific for the autophagy-related ubiquitin-like proteins GATE-16, MAP1-LC3, GABARAP, and Apg8L. J Biol Chem 2003, 278(51):51841-51850.

30. Tanida I, Tanida-Miyake E, Komatsu M, Ueno T, Kominami E: Human Apg3p/ Aut1p homologue is an authentic E2 enzyme for multiple substrates, GATE-16, GABARAP, and MAP-LC3, and facilitates the conjugation of hApg12p to hApg5p. J Biol Chem 2002, 277(16):13739-13744.

31. Tanida I, Sou Y, Minematsu-lkeguchi N, Ueno T, Kominami E: Atg8L/Apg8L is the fourth mammalian modifier of mammalian Atg8 conjugation mediated by human Atg4B, Atg7 and Atg3. FEBS J 2006, 273(11):2553-2562.

32. Hiller M, Huse K, Szafranski K, Jahn N, Hampe J, Schreiber S, Backofen R, Platzer M: Widespread occurrence of alternative splicing at NAGNAG 
acceptors contributes to proteome plasticity. Nat Genet 2004, 36(12):1255-1257.

33. Modrek B, Lee C: A genomic view of alternative splicing. Nat Genet 2002, 30(1):13-19.

34. Koscielny G, Le Texier V, Gopalakrishnan C, Kumanduri V, Riethoven JJ, Nardone F, Stanley E, Fallsehr C, Hofmann O, Kull M, et al: ASTD: The Alternative Splicing and Transcript Diversity database. Genomics 2009, 93(3):213-220.

35. Tanida I, Ueno T, Kominami E: Human light chain 3/MAP1LC3B is cleaved at its carboxyl-terminal Met121 to expose Gly120 for lipidation and targeting to autophagosomal membranes. J Biol Chem 2004 279(46):47704-47710.

36. Pankiv S, Hoyvarade Clausen T, Lamark T, Brech A, Bruun JA, Outzen H, Overvatn A, Bjorkoy G, Johansen T: p62/SGSTM1 binds directly to Atg8/ LC3 to facilitate degradation of ubiquitinated protein aggregates by autophagy. J Biol Chem 2007, 282(33):24131-24145.

37. Kumeta $\mathrm{H}$, Watanabe $M$, Nakatogawa $H$, Yamaguchi M, Ogura K, Adachi W, Fujioka $Y$, Noda NN, Ohsumi $Y$, Inagaki F: The NMR structure of the autophagy-related protein Atg8. J Biomol NMR 2010, 47(3):237-241.

38. Sugawara K, Suzuki NN, Fujioka Y, Mizushima N, Ohsumi Y, Inagaki F: The crystal structure of microtubule-associated protein light chain 3, a mammalian homologue of Saccharomyces cerevisiae Atg8. Genes Cells 2004, 9(7):611-618

39. Coyle JE, Qamar S, Rajashankar KR, Nikolov DB: Structure of GABARAP in two conformations: implications for $\mathrm{GABA}(\mathrm{A})$ receptor localization and tubulin binding. Neuron 2002, 33(1):63-74.

40. Bavro VN, Sola M, Bracher A, Kneussel M, Betz H, Weissenhorn W: Crystal structure of the GABAA-receptor-associated protein, GABARAP. EMBO Rep 2002, 3:183-189.

41. Paz Y, Elazar Z, Fass D: Structure of GATE-16, membrane transport modulator and mammalian ortholog of autophagocytosis factor Aut7p. J Biol Chem 2000, 275(33):25445-25450

42. Satoo K, Suzuki NN, Fujioka Y, Mizushima N, Ohsumi Y, Inagaki F: Crystallization and preliminary crystallographic analysis of human Atg4B-LC3 complex. Acta Crystallogr Sect F Struct Biol Cryst Commun 2007, 63(2):99-102.

43. Black DL: Mechanisms of alternative pre-messenger RNA splicing. Annu Rev Biochem 2003, 72:291-336.

44. Condorelli GBR, Smith RJ: Two alternatively spliced forms of the human insulin-like growth factor I receptor have distinct biological activities and internalization kinetics. J Biol Chem 1994, 269(11):8510-8516.

45. Tadokoro KY-IM, Tachibana M, Fujishiro M, Nagao K, Toyoda M, Ozaki M, Ono M, Miki N, Miyashita T, Yamada M: Frequent occurrence of protein isoforms with or without a single amino acid residue by subtle alternative splicing: the case of Gln in DRPLA affects subcellular localization of the products. J Hum Genet 2005, 50:382-394.

46. Vogan KJ, Underhill DA, Gros P: An alternative splicing event in the Pax-3 paired domain identifies the linker region as a key determinant of paired domain DNA-binding activity. Mol Cell Biol 1996, 16(12):6677-6686.

47. Lorkovic ZJ, Lehner R, Forstner C, Barta A: Evolutionary conservation of minor U12-type spliceosome between plants and humans. RNA 2005, 11(7):1095-1107.

48. Noda NN, Kumeta H, Nakatogawa H, Satoo K, Adachi W, Ishii J, Fujioka Y, Ohsumi Y, Inagaki F: Structural basis of target recognition by Atg8/LC3 during selective autophagy. Genes Cells 2008, 13(12):1211-1218.

49. Amar N, Lustig G, Ichimura Y, Ohsumi Y, Elazar Z: Two newly identified sites in the ubiquitin-like protein Atg8 are essential for autophagy. EMBO Rep 2006, 7(6):635-642.

50. Fass E, Amar N, Elazar Z: Identification of essential residues for the C-terminal cleavage of the mammalian LC3: a lesson from yeast Atg8. Autophagy 2007, 3(1):48.

51. Simsek M, Al-Bulushi T, Shanmugakonar M, Al-Barwani HS, Bayoumi R. Allele-specific amplification of exon 7 in the survival motor neuron (SMN) genes for molecular diagnosis of spinal muscular atrophy. Genet Test 2003, 7(4):325-327.

52. Schwede T, Kopp J, Guex N, Peitsch MC: SWISS-MODEL: An automated protein homology-modeling server. Nucleic Acids Res 2003, 31(13):3381-3385.

53. Kaminski G, Friesner RA, Tirado-Rives J, Jorgensen WL: Evaluation and reparametrization of the OPLS-AA force field for proteins via comparison with accurate quantum chemical calculations on peptides. $J$ Phys Chem $B$ 2001, 105(28):6474-6487.

54. Jorgensen WL: OPLS Force Fields. In The Encyclopedia of Computational Chemistry, Volume 3. Edited by Schleyer PR. New York: John Wiley \& Sons; 1998:1986-1989.

55. Berendsen HJC, van der Spoel D, van Drunen R: GROMACS: A messagepassing parallel molecular dynamics implementation. Comp Phys Comm 1995, 91:43-56.

56. Lindahl E, Hess B, van der Spoel D: GROMACS 3.0: a package for molecular simulation and trajectory analysis. J Mol Model 2001, 7:306-317.

57. Berendsen HJC, Postma JPM, Gunsteren WF, DiNola A, Haak JR: Molecular dynamics with coupling to an external bath. J Chem Phys 1984, 81:3684-3690

58. DeLano WL: The PyMOL Molecular Graphics System. San Carlos, CA: DeLano Scientific LLC; 2004.

doi:10.1186/1471-2121-14-27

Cite this article as: Liu et al: Arginine68 is an essential residue for the C-terminal cleavage of human Atg8 family proteins. BMC Cell Biology 2013 14:27.

\section{Submit your next manuscript to BioMed Central and take full advantage of:}

- Convenient online submission

- Thorough peer review

- No space constraints or color figure charges

- Immediate publication on acceptance

- Inclusion in PubMed, CAS, Scopus and Google Scholar

- Research which is freely available for redistribution 